\title{
Mobility-Aware Video Streaming in MIMO-Capable Heterogeneous Wireless Networks
}

\author{
Hayoung Oh \\ School of DASAN University College, Ajou University, Suwon, Gyeonggi, Republic of Korea \\ Correspondence should be addressed to Hayoung Oh; hyoh@ssu.ac.kr
}

Received 18 March 2016; Revised 15 June 2016; Accepted 19 July 2016

Academic Editor: Zhao Cheng

Copyright (C) 2016 Hayoung Oh. This is an open access article distributed under the Creative Commons Attribution License, which permits unrestricted use, distribution, and reproduction in any medium, provided the original work is properly cited.

\begin{abstract}
Multiple input and multiple output (MIMO) is a well-known technique for the exploitation of the spatial multiplexing (MUX) and spatial diversity (DIV) gains that improve transmission quality and reliability. In this paper, we propose a quality-adaptive scheme for handover and forwarding that supports mobile-video-streaming services in MIMO-capable, heterogeneous wirelessaccess networks such as those for Wi-Fi and LTE. Unlike previous handover schemes, we propose an appropriate metric for the selection of the wireless technology and the MIMO mode, whereby a new address availability and the wireless-channel quality, both of which are in a new wireless-access network so that the handover and video-playing delays are reduced, are considered. While an MN maintains its original care-of address (oCoA), the video packets destined for the MN are forwarded with the MIMO technique (MUX mode or DIV mode) on top of a specific wireless technology from the previous Access Router (pAR) to the new Access Router (nAR) until they finally reach the MN; however, to guarantee a high video-streaming quality and to limit the videopacket-forwarding hops between the pAR and the nAR, the MN creates a new CoA (nCOA) within the delay threshold of the QoS/quality of experience (QoE) satisfaction result, and then, as much as possible, the video packet is forwarded with the MUX. Through extensive simulations, we show that the proposed scheme is a significant improvement upon the other schemes.
\end{abstract}

\section{Introduction}

Over recent years, "multiple input and multiple output" (MIMO) technology has grown rapidly in the field of wireless communications. MIMO links have provided a high spectral efficiency in wireless environments through multiple spatial channels and without a need for additional bandwidth requirements [1]. MIMO links provide the following two operational options: (a) simultaneous transmission of different data by multiple antennas that increases the data rate, called "spatial multiplexing" (MUX), and (b) simultaneous transmission of the same data by multiple antennas that increases the reliability or the transmission range, called "spatial diversity" (DIV). With the proliferation of MIMOcapable, heterogeneous wireless technologies and mobile electronic smart devices such as iPhones and iPads, there is a fast-growing interest in mobile video-streaming traffic for such mobile devices. According to the Cisco visualnetworking index, mobile video traffic will grow 66 -fold over a period of five years [2-4].
One of the main challenges regarding MIMO-capable, heterogeneous wireless technologies is the provision of the support for a robust mobile video service such as Internet Protocol television (IPTV), Voice over Internet Protocol (VoIP) [5], and video streaming [2, 3], whereby fast and seamless handover and forwarding schemes are supported between heterogeneous wireless-access networks. In this environment, seamless mobility and data forwarding are coupled according to user preferences, enabling mobile users to be "always best connected" (ABC) so that quality of service and quality of experience (QoS and QoE) are optimized and maintained; furthermore, the core network of the heterogeneous wireless-access networks continues to evolve into an all-IP-based network. Accordingly, to reduce the handover latency and solve the packet-loss problem, a fast MIPv6 (FMIPv6) handover [6] with an address preconfiguration and a fast IP connectivity has been proposed after the introduction of the Mobile IPv6 (MIPv6) [7] in the Internet Engineering Task Force (IETF); however, this handover proposal is still not robust for mobile video 
streaming in MIMO-capable, heterogeneous wireless technologies. Although Hierarchical MIPv6 (HMIPv6) [8] and Proxy Mobile IPv6 (PMIPv6) [9] have been proposed for location management and handover-latency enhancement, corresponding limits in the local mobility region persist; plus, even though the GPRS Tunneling Protocol (GTP) and the Dual Stack MIP (DSMIP) are proposed for the support of the IP mobility level between LTE and Wi-Fi, we have chosen to focus on FMIPv6 enhancement to cover the QoS and QoE of mobile video streaming, as well as the global mobility in MIMO-capable, heterogeneous wireless technologies.

Regarding FMIPv6, a mobile node $(\mathrm{MN})$ is previously configured with only one of the new care-of addresses (CoAs) of a specific wireless network before it is attached to a new link. This address preconfiguration, however, is useless when the $\mathrm{MN}$ moves to another visited network, or when the change of wireless technology by the $\mathrm{MN}$ is in contrast with the corresponding anticipation; therefore, the selection of an inappropriate wireless technology occurs, leading to a fluctuating mobile video-streaming quality that is due to a lack of unused new addresses or an unreliable wirelesschannel quality. In this case, FMIPv6 again follows the handover procedure of MIPv6 so that the handover latency increases undesirably. Attempts to improve both MIPv6 and FMIPv6 in a wireless network under the strong assumption of a perfect anticipation have already been completed.

Different from the previous works [10-13], specifically, the main goal of our proposed scheme is a mechanism that selects the "best," according to an appropriate metric, wireless technology for a robust mobile-video-streaming service among all of the wireless technologies, whereby the handover and video latency are reduced on average, and the effects of perfect and imperfect handover predictions are analyzed in terms of MIMO-capable, heterogeneous wireless technologies.

To our knowledge, this is the first report regarding the problem concerning the need for robust mobile video streaming in terms of MIMO-capable, heterogeneous wireless technologies. Unlike FMIPv6, for which the preconfiguration of one tentative $\mathrm{CoA}$ at an MN handover is used, two of the verified tentative CoAs from heterogeneous wirelessaccess networks are exploited for the proposed scheme to prevent a fast handover from failing with any FMIPv6-related wireless technology for which there is no provisional CoA in a new visited network. By making a fast handover possible in any situation, the scheme reduces the average handover latency and the mobile-video-streaming delay by using a MIMO-transmission mode. We have therefore defined a metric that captures the contribution that each wireless technology provides for the quality improvement of mobile video streaming, while also considering the importance of video packets (in terms of video distortion and play-out deadlines), the QoE, and the video-packet-forwarding mode of MIMO technologies such as MUX and DIV with respect to MIMO-capable, heterogeneous wireless technologies.

Through a performance evaluation, we show that our scheme provides a mechanism that is more robust than those of the other schemes such as eFMIPv6 [10], FMIPv6, HMIPv6, the hybrid algorithm [14] and Handoff Protocol for
Integrated Networks (HPINs) [15] for which the MIMO routing protocol (MIR) [16] of mobile-video-streaming services is considered.

The rest of this paper is organized as follows: We present related work and the general system architecture for the proposed scheme in Sections 2 and 3; regarding the MIMO-capable heterogeneous wireless technologies, a robust mobile-video-streaming scheme is described in Section 4; Section 5 presents the analysis and performance results; and lastly, we conclude the paper in Section 6.

\section{Related Work}

The authors of [17] propose a simulation platform for the analysis of the handover issue for downlink coordinated multipoint (CoMP) transmissions in LTE-A cellular systems. Among the variety of intercell interference coordination (ICIC) strategies, the authors applied a frequency-reuse factor of one in the cell-center areas and a higher reuse factor in the cell-edge areas. In [18], the authors propose a downlink soft handover scheme for cell-edge users in 4G-LTE systems to improve the cell-edge capacity. In the cell-edge areas, capacity loss generally exists; therefore, the authors of [18] used a multicell MIMO, with the cooperation of multiple base stations and different, adaptively exploited multi-cell MIMO transmissions, based on the spatial correlation of a downlink channel and the path loss of adjacent cells, whereby the ergodic link-level capacities are compared. In [19], the authors proposed a novel handover scheme for which the number of detected antennas in a MIMO-capable wireless communication system is used; through the diminishing of the MAC-feedback quantity in the base station, the scheme results in a diversification of the handover decision and a reduction of the MAC overhead. In [20], the authors introduce handover sequences by consisting of a comb cyclically shifted in the frequency domain to identify the MIMO cells. Orthogonal sequences in time domain are used to identify the antennas of MIMO within a cell. Sequence assignment to the cells follows a classical frequency-reuse scheme. With these sequences, the frequency-selective multicell channel of MIMO can be identified with high precision also at the cell edge. In [21], the authors carried out the research of influence by the multiantenna technology MIMO on realization of handover procedure in cellular radio access systems. By means of modeling possibilities, the authors showed both improvements and deterioration of duration of handover procedure with the use of MIMO in radio systems. In [22], the authors consider a novel adaptive multi-input multioutput (MIMO) semisoft handover technique for the quality of service (QoS). Semisoft handover permits both hard and soft handover advantages for OFDM networks. Specifically, they analyze the semisoft handover combined with the adaptive MIMO mode switching scheme. In [23], the authors implement the Variable Step Size Griffiths (VSSG) algorithm for steering the radiation pattern of the eNodeB with multiple input multiple output (MIMO) antenna's from -90 degrees to +90 degrees. After steering the main beam, the detection is performed using MUSIC algorithm for detecting the UE's located at cell boundaries during handover to 
provide better signal strength to meet the standards QoS of 4G-LTE. They also compared with capacity of single input single output (SISO) and multiple input multiple output (MIMO). However, above schemes mainly focus on validating the effectiveness of adaptive MIMO soft handover in the femtocell or cell edge networks or showing that the influence of the correlation between the signal qualities of the source and target base station (BS). In addition, they did not consider MIMO and video streaming.

In [14], the authors investigated the potentiality and benefits of a novel vertical-handover algorithm for which both hard and soft handovers are exploited in a dual-mode configuration, and it was compared with the traditional hard approach. Regarding the hard vertical-handover mechanism, the connectivity between the mobile users and the serving network is broken before the connection with a new network is established (namely, "break-before-make"); alternatively, the soft vertical-handover mechanism is "make-beforebreak" and generally improves the seamless connectivity. The algorithm of [14] aims to maintain seamless connectivity for those users moving in heterogeneous-network environments (i.e., comprised of WLAN hotspots and UMTS base stations), while still guaranteeing the user-QoS requirements. Notably, though, a fully forwarding MN scheme that is based on the MIMO mode from the pAR to the nAR in terms of the handover was not considered for these schemes; furthermore, the selection metric of the wireless technique was not simultaneously considered.

The authors of [24] propose a cross-layer and reactive handover procedure which employs optimized movement detection and address configuration schemes based on the standard specification for HMIPv6 mobility support. For that, they utilize the advantage of link layer notification in the link layer of an AP's protocol stack and the network layer of the AR which has a connection with the AP. However, their assumption that an AP knows the exact AR to which it is attached is strict nowadays and they did not consider MIMO and video streaming.

In [25], the authors present a data rate-guaranteed IP mobility management scheme for fast-moving vehicles with multiple wireless network interfaces. Different from other previous work, they assume the handover initiation is based on the measured data rate rather than the radio signal strength. To guarantee the required data rate, they consider multiple bidirectional IP tunnels locally constructed between the HMIPv6 MAP and the mobile gateway (MG). The packets are distributed in parallel over these tunnels during handover operation, while eliminating the possible delay and packet loss during handover operation. However, they consider not video streaming with MIMO but the UDP-based audio application traffic for the type of the application traffic.

In [26], the authors investigate the potential of applying FMIPv6 in vehicular environments by using IEEE 802.21 Media Independent Handover (MIH) services. With the aid of the lower three layers' information of the MIH enabled $\mathrm{MN} / \mathrm{MR}$ and the neighboring access networks they design an "Information Element Container" to store static and dynamic L2 and L3 information of neighboring access networks. Plus, they propose a special cache maintained by the MN/MR to reduce the anticipation time in FMIPv6, thus increasing the probability of the predictive mode of operation for a cross-layer mechanism. The lower layer information of the available links obtained by MIH services and the higher layer information such as QoS parameter requirements of the applications are used by a Policy Engine (PE) to make intelligent handover decisions. However, they only consider a network scenario, where one WiMAX (IEEE 802.16) cell and one IEEE 802.11b WLAN Basic Service Set (BSS) are located based on one mobility service provider. Also, they did not consider video streaming with MIMO for the type of the application traffic.

In [27], the authors focus on mobility management at a convergent layer for heterogeneous networks, network layer with mobile Internet Protocols (MIPs), to support VoIP services in wireless heterogeneous networks. They identify four crucial parameters that affect the handover performances of the protocols, depending on the FER in the air link. These are the number of messages exchanged over the air link, the entities involved in the process, the retransmission strategy (maximum number of retransmissions allowed, back-off mechanism, and back-off timer), and the message sizes of the protocols. With one of them, to optimize the handover delay, the authors propose to use the adaptive retransmission proportional to the size of the messages involved in the transactions of the handover process. However, their scheme is not practical for mobile video streaming since the support of VoIP in mobile systems requires low handover latency (i.e., $<400 \mathrm{~ms}$ ) to achieve seamless handovers. Plus, they only focus on network layer handover; therefore, they do not consider link layer detection and retransmissions as well as the impact of correlated errors on the disruption time, error correction mechanisms, processing, and queuing delays.

In [15], the authors proposes a novel architecture called Integrated InterSystem Architecture (IISA) based on 3GPP/3GPP2-WLAN interworking models to permit the integration of any type of wireless networks. Furthermore, they propose a mobility management scheme called the Handoff Protocol for Integrated Networks (HPINs), which provides QoS guarantees for real-time applications of mobile users moving across various networks. HPIN allows the selection of the best available network at any given time, for both heterogeneous and homogeneous wireless networks. However, they assume a third-party entity called the interworking decision engine (IDE) to guarantee the seamless roaming and service continuity required in $4 \mathrm{G} / \mathrm{NGWNs}$. The routers extract QoS context information, and according to the context received, the intermediate router reserves corresponding resources and updates the path information.

\section{System Architecture}

Generally, regarding MIMO-capable, heterogeneous wireless technologies, several heterogeneous wireless networks can coexist; moreover, the Internet serves as a backbone that connects a home network and several heterogeneous visited networks including Wi-Fi and LTE. The home network is where the global IPv6 address (home address) of an MN 
exists. The IPv6 address that is based on the 48-bit MAC address of the MN is 128 bits and consists of the AR prefix (64 bits) and the Modified EUI-64. The home address is a unicast routable address that has been assigned to the $\mathrm{MN}$ and is used as the permanent MN address. Standard IP-routing mechanisms will deliver packets that are destined for an MNhome address. The domain of a visited network comprises several ARs and wireless APs that an MN can connect with [10]. We assume that each AR comprises an interface that is connected to a distinct set of APs and that the same network prefix cannot be assigned to the interface of a different AR; that is, ARs are distinguished by their own prefix. Generally, a CoA can be used to enable an MN so that it can send and receive packets to a Home Agent (HA) or a Correspondent Node $(\mathrm{CN})$; the HA is a router in the MN-home network that the $\mathrm{MN}$ has registered its CoA with. While the $\mathrm{MN}$ is away from the home network, the HA intercepts the packets that are destined for the MN-home address, encapsulates them, and tunnels them to the MN's registered CoA; furthermore, the association between an MN's home address and a CoA is known as a "binding" for the MN. While away from the home network, an $\mathrm{MN}$ registers its new $\mathrm{CoA}$ (nCoA) with a home address, whereby the $\mathrm{MN}$ performs a binding registration by sending a binding update message to the HA; the HA reply to the $\mathrm{MN}$ is in the form of a Binding Acknowledgement message. The $\mathrm{CN}$ that can be either mobile or stationary is a peer node that the MN communicates with. The nCoA that is composed of the nAR prefix in a new visited network, and the MAC address of the MN is a unicast routable address that is associated with an MN while a new visited network is being visited. The $\mathrm{nCoA}$ is made after the Duplicate Address Detection (DAD) is completed. The DAD corresponds to most of the handover latency because it requires time in the order of seconds to detect whether the $\mathrm{nCoA}$ of the $\mathrm{MN}$ has been duplicated. Different from [10], the MN in this paper sophisticatedly selects the best wireless technology from among multiple wireless network interfaces (WNICs) and a MIMO forwarding scheme for robust mobile video streaming.

Parallel to the advances of heterogeneous wireless technologies and MIMO modes is the development of a robust mobile-video-streaming paradigm [2-4]; thus, we consider mobile video streaming over MIMO-capable, heterogeneous wireless networks where the AP can forward video packets to other APs with a combination of both the wireless network (i.e., Wi-Fi or LTE) and the MIMO transmission mode (i.e., MUX or DIV) based on the distance and channel quality. Similarly, through an inspection of the relation between the $\mathrm{AP}$ and the $\mathrm{MN}$, the $\mathrm{MN}$ can also select the wireless technology (i.e., Wi-Fi or LTE) and the MIMO-transmission mode for a robust mobile-video-streaming service. To cover a general pair of wireless networks comprising the MIMO mode and the handover possibility, we designed an appropriate selection metric using the QoS and the QoE.

Based on the extensive evaluations, we obtained the following frequently used pair: a Wi-Fi and MUX mixture is generally used between the AP and MN over a reliable wireless link. Plus, an LTE and DIV mixture is generally used between the oAP and $\mathrm{nAP}$ due to the limit regarding the distance and channel quality; however, both cases can only be guaranteed where there is a possibility that the $\mathrm{nCoA}$ can be used for a successful handover. We will explain the proposed metric in terms of the covering of the $\mathrm{nCoA}$ resource management, whereby the wireless techniques and the MIMO modes are selected.

\section{A Robust Mobile-Video-Streaming Scheme for MIMO-Capable Heterogeneous Networks}

In this section, we provide the details of the proposed scheme, whereby an appropriate wireless-technology metric, the MIMO mode, and the temporal reuse of verified tentative CoAs are selected for robust mobile video streaming in MIMO-capable, heterogeneous wireless networks, along with a depiction of the architectural view in Figure 1. For robust mobile video streaming, the $\mathrm{MN}$ of the previous visited network selects the "best," according to an appropriate metric, new visited network among all of the wireless technologies and a MIMO mode for the seamless handover whereby a tentative address management is proactively performed by the ARs. For an appropriate metric for the video quality of an encoded sequence, we used the average peak signalto-noise ratio (PSNR) [28, 29] (i.e., the PSNR based on the luminance $(Y)$ component of video sequences) that is measured in $\mathrm{dB}$ and averaged it over the entire duration of the video sequence. For the QoE, we applied the concept of a "temporal quality assessment" [30], whereby the difference of the corresponding pixel values in the two consecutive neighboring frames is first measured to estimate the motion of the objects in the video.

4.1. Metric Selection of the Wireless Technology and the Forwarding Scheme. To choose the "best" wireless technology, a metric that captures the contribution of each wireless technology regarding the mobile-video-quality improvement needs to be defined first with the system state. Let $Q_{w_{i}}\left(c_{j}\right)$ be the improvement of the mobile-video quality and the QoE at the $\operatorname{MN}\left(c_{j}\right)$ when the wireless technology $w_{i}$ is selected, as follows:

$$
Q_{w_{i}}\left(c_{j}\right)=\sum_{p=1}^{N}\left(1-e_{p, w_{i}}\right) \cdot I_{p, w_{i}, m_{i}} \cdot \mathrm{TVM}_{p} \cdot R_{p, w_{i}},
$$

where $p$ means each video frame, $N$ is the total video frames per a group of pictures (GoP) that is included in the $\operatorname{MN}\left(c_{j}\right)$, and $e_{p, w_{i}}$ is the loss probability of the frame $p$ in the wireless technology $w_{i}$ that is due to latency or channel errors. $e_{p, w_{i}}$ is given by the following:

$$
\begin{aligned}
e_{p, w_{i}}= & \int_{\tau_{w_{i}}}^{\infty} f\left(t, w_{i}\right) d t+\left(1-\int_{\tau_{w_{i}}}^{\infty} f\left(t, w_{i}\right) d t\right) \\
& \cdot f(s)
\end{aligned}
$$

In (2), the first part describes the probability of the late arrival of a video frame, and $\tau_{w_{i}}$ and $f\left(t, w_{i}\right)$ are the remaining 


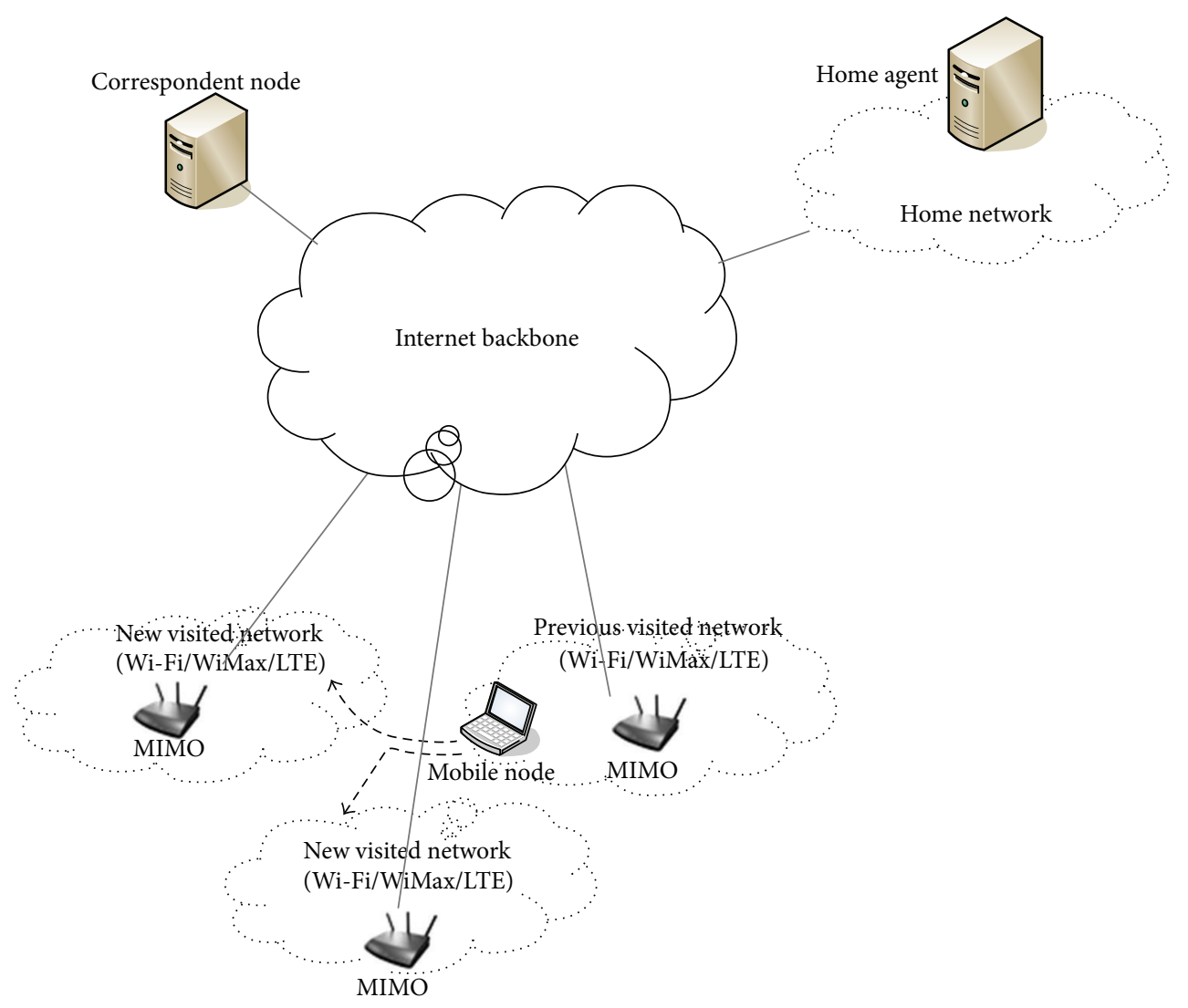

FIGURE 1: Architectural view of the proposed scheme.

time until the playout deadline and the distribution of the forward trip time in $w_{i}$, respectively. The late-arrival probability of a frame corresponds with the following framedrop probability:

$$
\begin{aligned}
\int_{\tau_{w_{i}}}^{\infty} f\left(t, w_{i}\right) d t & =1-\int_{-\infty}^{\tau_{w_{i}}} f\left(t, w_{i}\right) d t \\
& =1-P\left(X \leq \tau_{w_{i}}\right) \\
& =1-\left(1-e^{-(1 / 4) \tau_{w_{i}}}\right)=e^{-(1 / 4) \tau_{w_{i}}} .
\end{aligned}
$$

The second part of (2) describes the loss probability (of a frame that is still on time) that is due to the effects of the wireless channel such as noise, fading, and interference; meanwhile, $f(s)$ is a snapshot of the loss probability per each state according to the channel characteristics of Wi-Fi or LTE. The states will be explained in detail during the presentation of the random-mobility model.

Intuitively, mobility causes glitches and stalls of mobile video, and it also causes fast, unpredictable variations of the channel quality that are shown Figure 3. In the case of Wi$\mathrm{Fi}$, it is only possible for an $\mathrm{MN}$ to use mobility within a specific transmission range. For that, we also utilize Figures 4 and 5 of [11-13]. However, different from Wi-Fi case similar with the previous works [11-13], in this paper we extend LTE case as well as Wi-Fi as shown in Figure 7. Figure 4 presents the next four MN states according to the initial MN state
$(0,0)$ and without the MN speed, in a random-walk model. Figure 5 shows all of the nine possible states that are based only on the $\mathrm{MN}$ locations according to the random-mobility model. Although the Wi-Fi signal can reach the end of the transmission range, the reception probability is decreased, as shown in Figure 2, because the signal strength is very weak in the boundary of the transmission range; therefore, considering the $\delta$ effect shown in Figure 6, it is better for the $\mathrm{MN}$ to use other wireless technologies instead of Wi-Fi when the distance between the AP and the mobile $\mathrm{MN}$ is larger than $40 \mathrm{~m}$.

If, however, we consider both the direction and the speed of the $\mathrm{MN}$ for the random-walk model, the mobile MN should naturally use LTE instead of Wi-Fi for seamless video streaming, as shown in Figure 7; moreover, the possible states can be extended, as shown in Figure 8, with three indexes. The circles indicate the Wi-Fi zones that are expressed by the state " $x, y, 0$ " with a low MN speed, whereas if the MN moves fast toward the cloud boundary with the middle speed, the state " $x, y, 1$ " that represents the Wi-Fi-boundary zone is used. The outlier of the cloud, expressed by the state " $x, y, 2$," is for LTE and the corresponding quick attainment of a high mobility. Lastly, our systems can be expressed with 27 states (" $x, y, z$ ") along with the direction and speed of the MN for both Wi$\mathrm{Fi}$ and LTE. Based on the direction and speed, (1) is used for the selection of a wireless technology by the MN according to the priority that is deemed by the order (i.e., Wi-Fi and LTE) found at the initial location. 


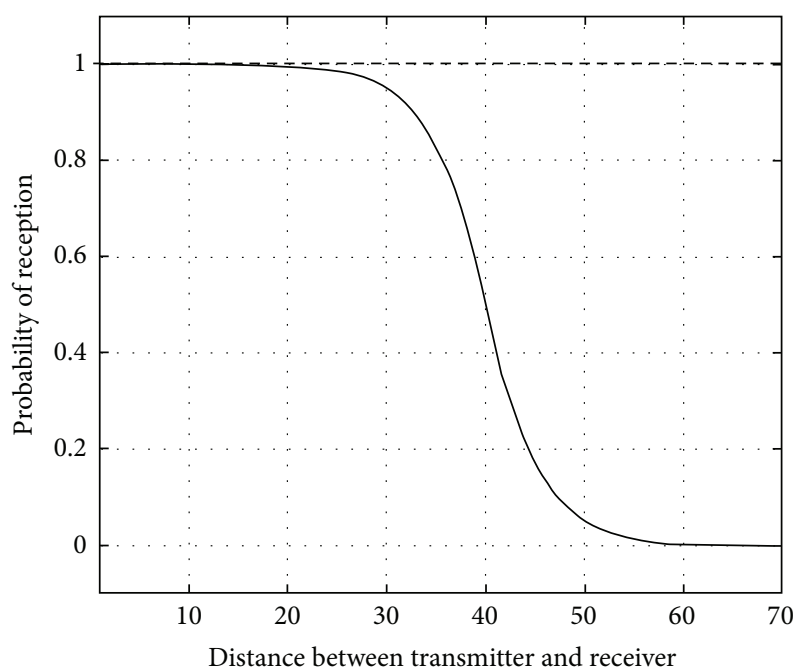

- Wi-Fi

- - LTE

Figure 2: Reception probability under a typical fading model.

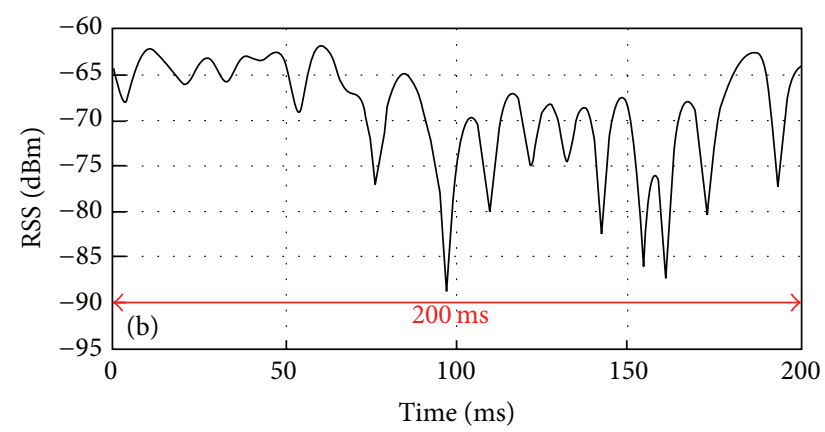

Figure 3: Unpredictable variations of channel quality due to random MN mobility.

$I_{p, w_{i}, m_{i}}$ represents the improvement of the mobile-video quality, where the standard metric of video quality, PSNR, is used, if a frame $p$ is received correctly and on time at the wireless technology $w_{i}$, and is in consideration of the MIMOtechnique mode $m_{i}$ between the MUX and the DIV. The PSNR is calculated on the frame and is most easily defined via the mean squared error (MSE), when the following noisefree $m \times n$ monochrome image $I$ and its noisy approximation $K$ are given:

$$
\begin{aligned}
& \operatorname{PSNR}(\mathrm{dB})=10 \cdot \log _{10}\left(\frac{\mathrm{MAX}_{I}^{2}}{\mathrm{MSE}}\right) \\
& =10 \\
& \quad \cdot \log _{10}\left(\frac{\operatorname{MAX}_{I}^{2}}{(1 / m n) \sum_{i=0}^{m-1} \sum_{j=0}^{n-1}[I(i, j)-K(i, j)]^{2}}\right) .
\end{aligned}
$$

Here, MAX $_{I}$ is the maximum pixel value of the frame that is possible.
To compute $I_{p, w_{i}, m_{i}}$, we decoded the entire video sequence with a packet that, according to $f(s)$, is missing and then computed the resultant distortion; plus, we recomputed $I_{p, w_{i}, m_{i}}$ based on the MIMO mode. We assume that this computation is performed offline at the $\operatorname{MN}\left(c_{j}\right)$ and that the distortion value is marked on each frame.

The DIV provides a long transmission possibility, and it improves the reliability of the wireless link for the same data with multiple antennas. As a result, even a disconnection scenario between two nodes in the DIV mode can form a well-designed forwarding method. In the case of MUX, the different data streams per each antenna can be transmitted between two nodes; therefore, if we use the MUX mode over reliable wireless links, the throughput can be improved very effectively.

For the proposed scheme, we opportunistically selected a MIMO forwarding scheme between the DIV and the MUX using (1); for example, in the following three scenarios, the DIV mode is selected:

(1) Over unreliable wireless link between the pAR and the mobile MN

(2) Far distance beween the pAR and the nAR

(3) Over unreliable wireless link between the nAR and the mobile $\mathrm{MN}$

Furthermore, for the following two scenarios, the MUX mode is selected:

(1) Over reliable wireless links between the pAR and the mobile MN

(2) Over reliable wireless links between the nAR and the mobile MN

$R_{p, w_{i}}$ is an indicator function that expresses whether it is possible for the wireless technology $w_{i}$ to service the mobile video frame $p$ with resources; the resources are the unused IP addresses that can be used for a temporal $\mathrm{CoA}_{w_{i}}$ by a visiting $M N$ (see Section 4.2). We define $R_{p, w_{i}}=1$ if the mobile video frame $p$ is serviced at the wireless technology $w_{i}$, or otherwise, it is defined as 0 .

$\mathrm{TVM}_{p}$ is calculated as the mean-square-value log of the difference between two consecutive frames $\left(F_{p-1}\right.$ and $\left.F_{p}\right)$ of the video (measured in $\mathrm{dB}$ ) for the $\mathrm{QoE}$ [30]. The wireless technology $w_{i}$ and the MIMO mode that maximizes the mobile video quality and the QoE improvement with the temporal CoA is therefore chosen for the proposed scheme. This is given with (5), as follows:

$$
\max _{w_{i}} Q_{w_{i}}\left(c_{j}\right)
$$

4.2. Tentative Address Management (TAM). Unlike the previous works [10-13], each Access Router (AR), such as the nAR and the $\mathrm{pAR}$, manages each tentative address pool containing the unused IP addresses that can be used as a temporal CoA by a visiting $\mathrm{MN}$ regarding the wireless technology $w_{i}$; these addresses are denoted by the verified tentative $\operatorname{CoAs}_{w_{i}}$ s. The AR ensures that the verified tentative $\operatorname{CoAs}_{w_{i}}$ s that are registered in the pool are not currently used by the other 


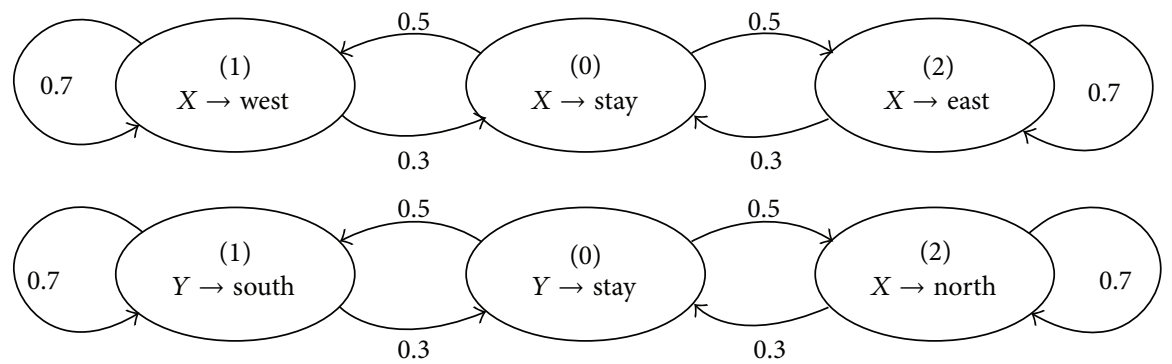

FIGURE 4: Flow chart of the probabilistic version of random-walk model.

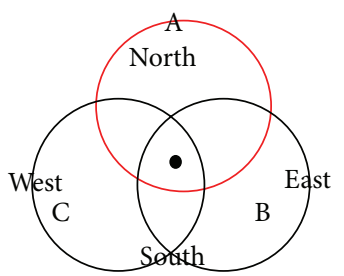

(1) State $(0,0)$

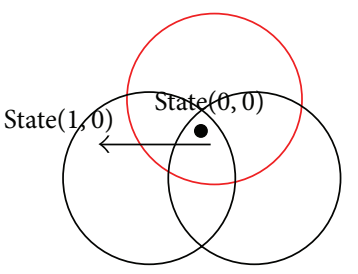

(4) $\operatorname{State}(1,0)$

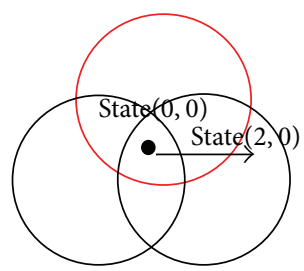

(7) $\operatorname{State}(2,0)$

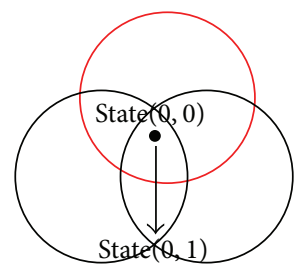

(2) $\operatorname{State}(0,1)$

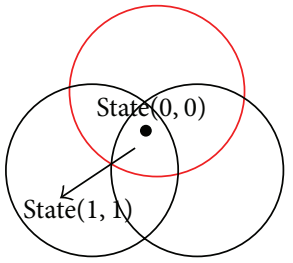

(5) State $(1,1)$

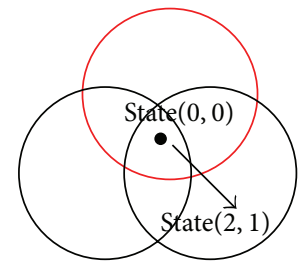

(8) $\operatorname{State}(2,1)$

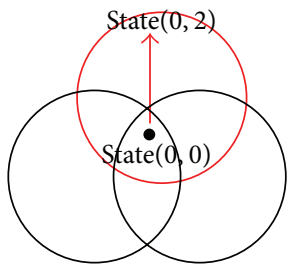

(3) State $(0,2)$

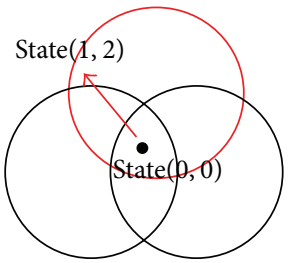

(6) State $(1,2)$

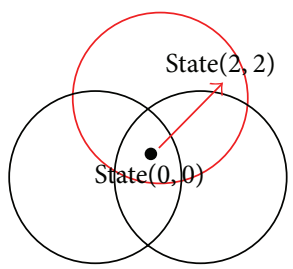

(9) State $(2,2)$

FIGURE 5: Possible next states of MN regarding random-walk model.

MNs by performing the DAD for each verified tentative address periodically. Basically, we assumed that each AR maintains as many of the verified tentative CoAs $w_{i}$ s according to the number of neighbor ARs in each of the possible wireless technologies. A verified tentative $\mathrm{CoA}_{w_{i}}$ is deleted from the pool if it is proven that it is being used by another node through the DAD. If the number of verified tentative $\mathrm{CoAs}_{w_{i}} \mathrm{~s}$ is smaller than the number of neighbor ARs, then the router adds new verified tentative $C o A s_{w}$ s into the pool by searching the available IP addresses using the already used MAC address of the other MNs and the AR prefix. In terms of the wireless technology $w_{i}$, we assumed that a modified Neighbor Router Advertisement $\left(\mathrm{mNRA}_{w_{i}}\right)$ including the several nonoverlapping verified tentative $\mathrm{CoAs}_{w_{i}}$ s can be periodically sent and received between each of the routers; as a result, each router of the wireless technology $w_{i}$ can manage the available verified tentative $\mathrm{CoAs}_{w_{i}}$ s per the wireless technology of the access networks of a one-hop neighbor. Generally, the NRA contains router information [7], and we modified the reserved field in the NRA to include the several verified tentative CoAs $s_{w_{i}}$ s. When the pAR receives the modified Router Solicitation for Proxy $\left(\mathrm{mRtSolPr} w_{w_{i}}\right)$ from the $\mathrm{MN}$, it informs the modified Proxy Router Advertisement $\left(m P r R t A d v_{w_{i}}\right)$ containing several verified tentative CoAs $s_{w_{i}} \mathrm{~s}$ about the neighboring access networks of the MN before it moves to one of the visited networks. The $\mathrm{MN}$ then sends a Last Packet (LP) message to the pAR informing it of the $\mathrm{MN}$ handover and moves to one of the new visited networks from the previous visited network. After the MN handover, the corresponding verified tentative $\mathrm{CoA}_{w_{i}}$ of the new visited network that is included in the modified Fast Neighbor Advertisement $\left(\mathrm{mFNA}_{w_{i}}\right)$ message is used by the 


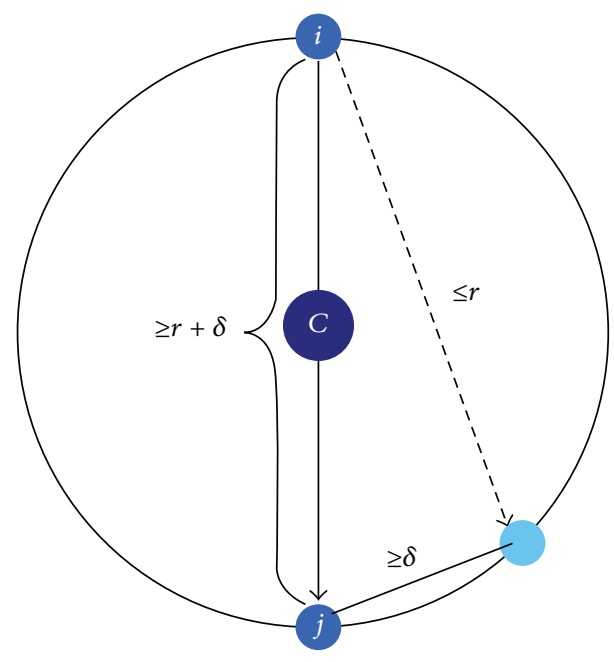

FIGURE 6: Distance relationship between the transmitter and a receiver.

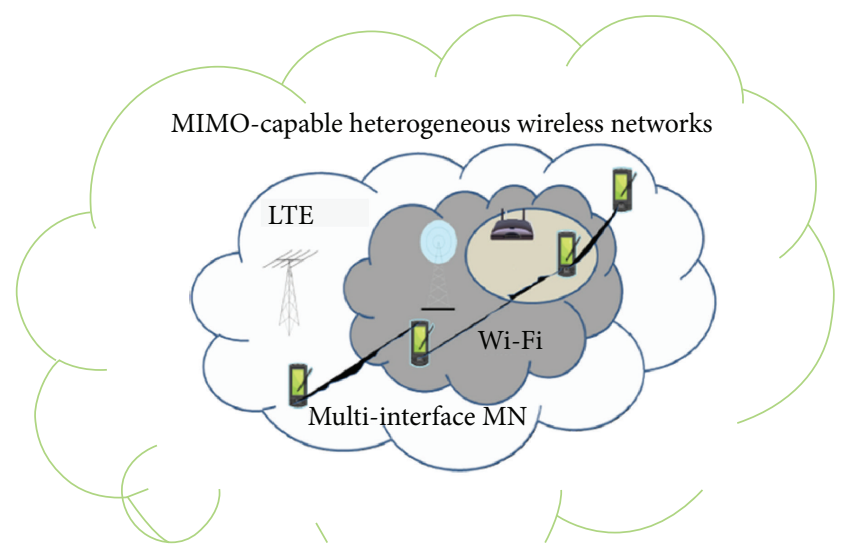

Figure 7: Mobile node (MN) seamlessly moving between different technologies.

$\mathrm{MN}$ to generate a fast IP connectivity that enables the $\mathrm{MN}$ to perform a binding update using the verified tentative $\mathrm{CoA}_{w_{i}}$, that is, the $\mathrm{FBU} 1_{w_{i}}$ and $\mathrm{BAcK} 1_{w_{i}}$. After the $\mathrm{MN}$ acquires its original $\mathrm{CoA}_{w_{i}}$ that contains its own MAC address by performing the DAD, the fast IP connectivity for which the verified tentative $\mathrm{CoA}_{w_{i}}$ is used is returned to the normal IP connectivity for which the original $\mathrm{CoA}_{w_{i}}$ is used. The recycling of the verified tentative $\mathrm{CoAs}_{w_{i}} \mathrm{~s}$ is possible because these verified tentative $\mathrm{CoAs}_{w_{i}} \mathrm{~s}$ are temporarily used by the MNs in the wireless technology $w_{i}$ for the reduction of the handover delay until the MN completes the binding updates through the use of its new original $\mathrm{CoA}_{w_{i}}$, that is, the $\mathrm{BU} 2_{w_{i}}$ and $\mathrm{BAcK} 2_{w_{i}}$. Figure 9 shows the handover procedure of the proposed scheme for mobile video streaming in terms of MIMO-capable, heterogeneous wireless networks; using L2layer triggers that are based on the MIH functionality [31], the MN detects mobility and the proposed metric of (1) is considered for seamless video streaming.

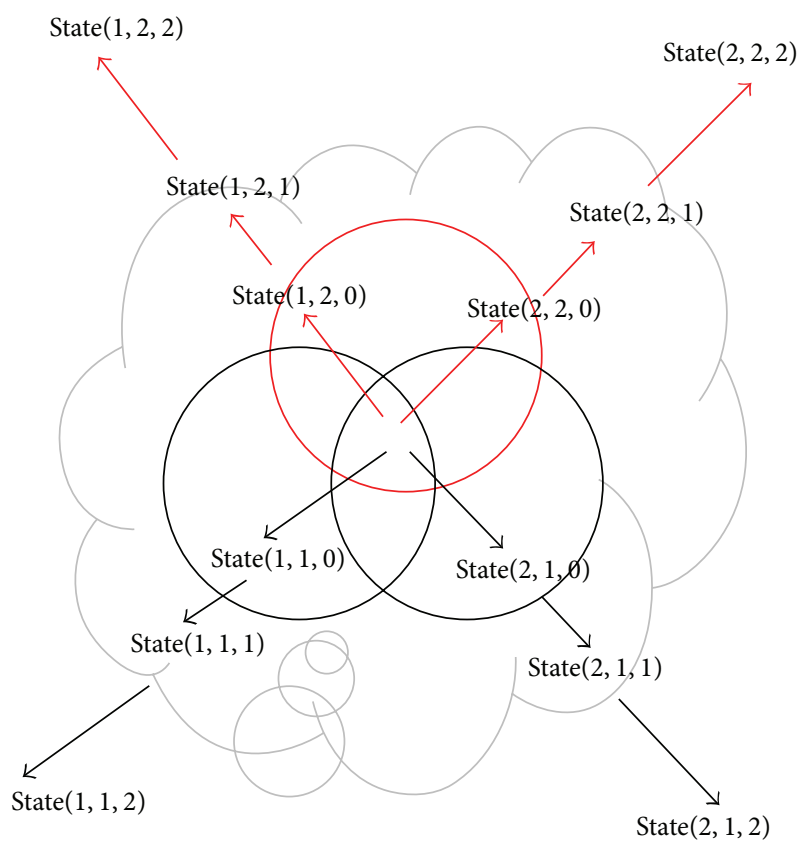

(1) State $(0,0,0)$

FIGURE 8: Extended random-walk model with the direction and speed of the MN.

\section{Performance Evaluation}

To evaluate the performances of the mobile video streaming of our proposed scheme and the other schemes, an NS3 simulation was performed with a variety of parameters (Table 1) such as coverage of AR, beacon interval, traffic type, and link delay. Figure 10 shows the network topology that was used in our simulation for which several entities including $\mathrm{HA}, \mathrm{CN}, \mathrm{AR}$, and $\mathrm{MN}$ were used. The link delay that contains propagation delay, processing delay, and queuing delay was assigned to $5 \mathrm{~ms}$ for the MN-AR links and the AR-Router links and $10 \mathrm{~ms}$ for the Router-CN/HA links and the ARAR links. The velocity at which the $\mathrm{MN}$ is moving across the network is up to $30 \mathrm{~m} / \mathrm{s}$. We used $2 \mathrm{Mbps}$ standard video traffic such as Carphone, Foreman, and Mother and Daughter [32] that are constantly sent from the CN to the MN. For the performance measures for the proposed scheme and the other schemes, we used the average PSNR for the video quality of an encoded sequence, the handover delay, and the video delay.

5.1. Handover Delay under Perfect Prediction. Figure 11 shows the video-streaming delay based on handover schemes such as MIPv6, HMIPv6, FMIPv6, eFMIPv6 [10], the hybrid scheme [14], HPIN [15] and the proposed scheme when the MNs move according to Figure 10. The video-streaming delay of the proposed scheme was prominently reduced and is therefore an improvement over the previous work [14]. This improvement is a direct result of the ability of the MN 


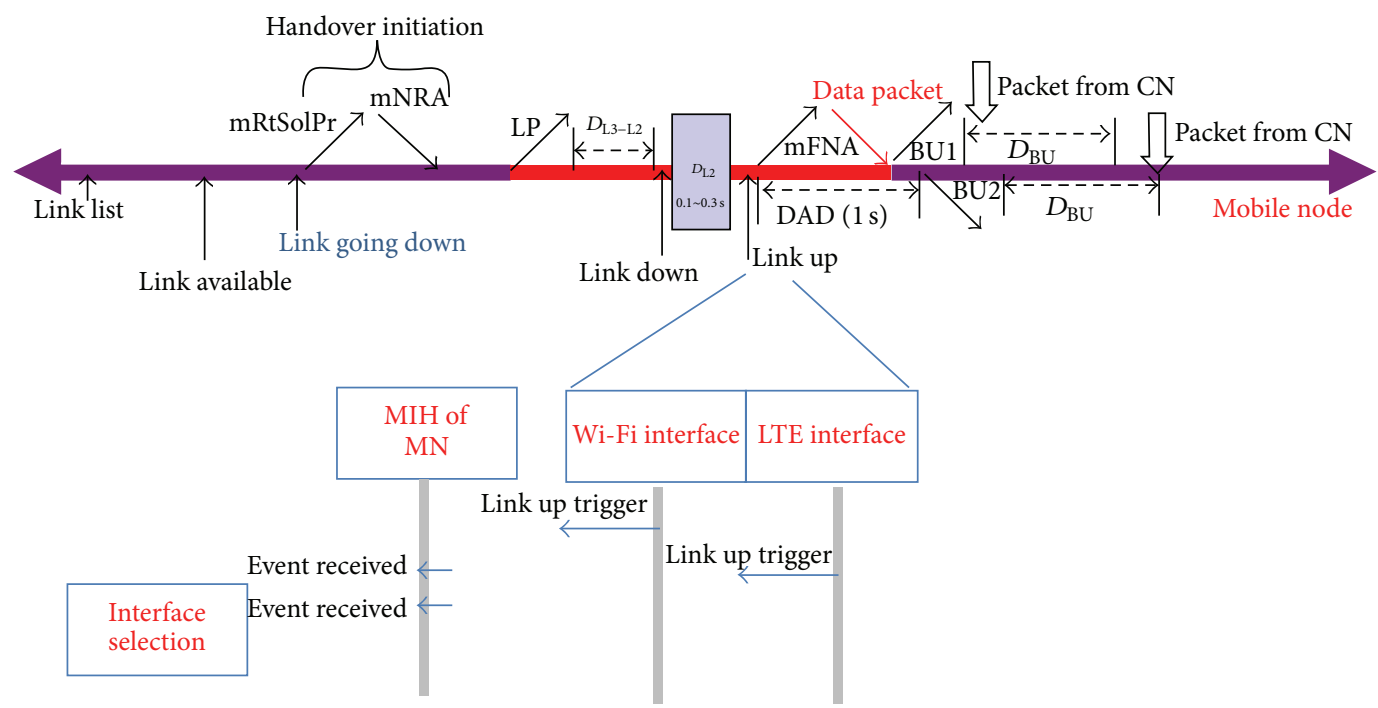

FIGURE 9: Handover procedure of the proposed scheme.

TABLE 1: Simulation properties.

\begin{tabular}{lc}
\hline Parameter & Value \\
\hline Coverage of AR & $120 \mathrm{~m}$ \\
Beacon interval & $0.1 \mathrm{~s}$ \\
Traffic type & $2 \mathrm{Mbps}$ video traffic \\
Link delay (MN-AR links and AR-Router links) & $5 \mathrm{~ms}$ \\
Link delay (Router-CN/HA links and AR-AR links) & $10 \mathrm{~ms}$ \\
Velocity & $1 \mathrm{~m} / \mathrm{s}$ to $15 \mathrm{~m} / \mathrm{s}$ \\
Channel model & Rayleigh fading \\
Simulation time & $180 \mathrm{~s}$ \\
Video sequence & Carphone, Foreman, Mother and Daughter \\
Radius of LTE and WLAN & $1000 \mathrm{~m}, 50 \mathrm{~m}$ \\
LTE and WLAN bandwidth & $10 \mathrm{MHz}, 20 \mathrm{MHz}$ \\
Frequency of WLAN & $2.412 \mathrm{GHz}$ \\
Tx power & $0.0134 \mathrm{~W}$ \\
Threshold (dBm) of LTE and WLAN & $-64,-60$ \\
\hline
\end{tabular}

whereby it can receive video packets from the pAR until the $\mathrm{MN}$ sends the LP to the $\mathrm{pAR}$, and the MN can also receive video packets as soon as it moves to new visited networks by using verified tentative CoAs that are based on the appropriate selection metric of the wireless technology with the MIMO-transmission mode; however, the Hierarchical MIPv6 (HMIPv6) [8] is proposed for the handover-latency enhancement in the local mobility region. We therefore focused on FMIPv6 enhancement in the proposed scheme to cover the global mobility as well as the local mobility. Even though the performance of HPIN [15] is very similar with the proposed scheme, the HPIN is not practical due the strong assumption of centralized scheme.

5.2. Handover Delay under Imperfect Prediction. To compare our proposed scheme with the hybrid scheme and eFMIPv6 in consideration of an imperfect handover prediction, we used the following mobility models: the random-walk model, the city section model, and the linear-walk model [33]. First, we analyzed and simulated the hybrid scheme, eFMIPv6, and our scheme using a random-walk model. It is useful to understand mobility patterns according to direction and speed, so unlike the previous work [10], we applied speed as well as direction for the random-walk model.

Figure 8 shows an example among a total of 27 states that are derived by the random-walk model whereby the combination of each $x, y$, and $z$ state is used. Each state represents the direction (north, south, east, west, north-east, north-west, south-east, south-west, and stay) and the speed.

We randomly located the MN in a part of the overlapping area of three circles, as shown in Figure 5, and this area is a common part of the link-going-down range of each of the visited networks. The MN randomly chooses one of the initial 27 states with the same probability, followed by its changing into one of the next states. Regarding Figure 5, we assumed that Area $\mathrm{A}$ is the pAR of the $\mathrm{MN}$ and that the $\mathrm{MN}$ immediately 


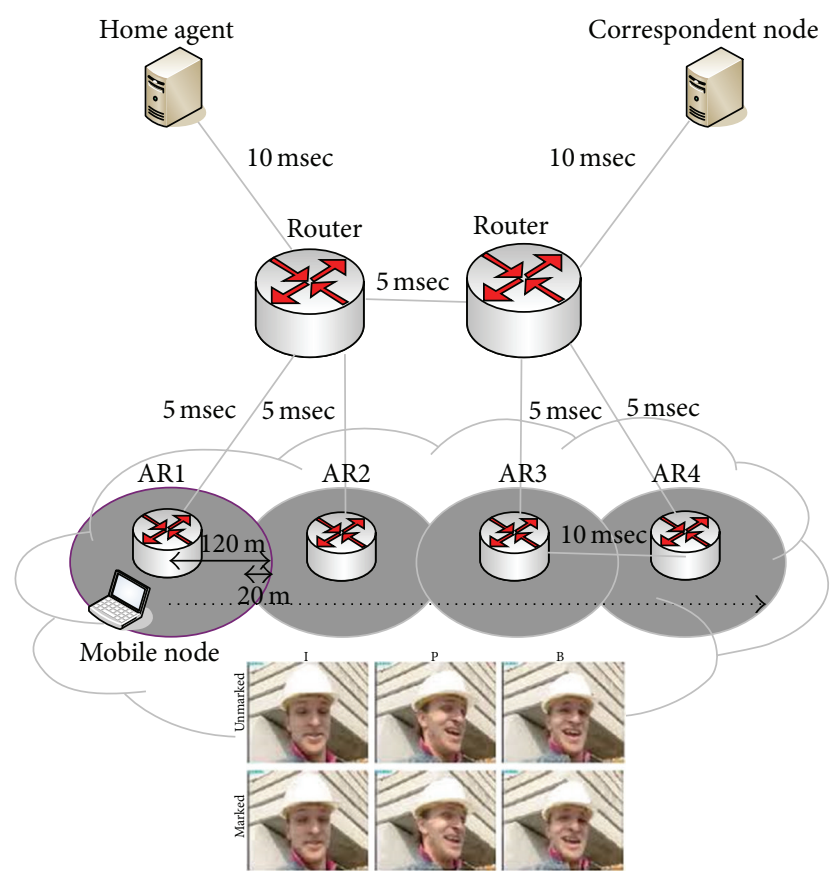

FIGURE 10: Simulation network topology.

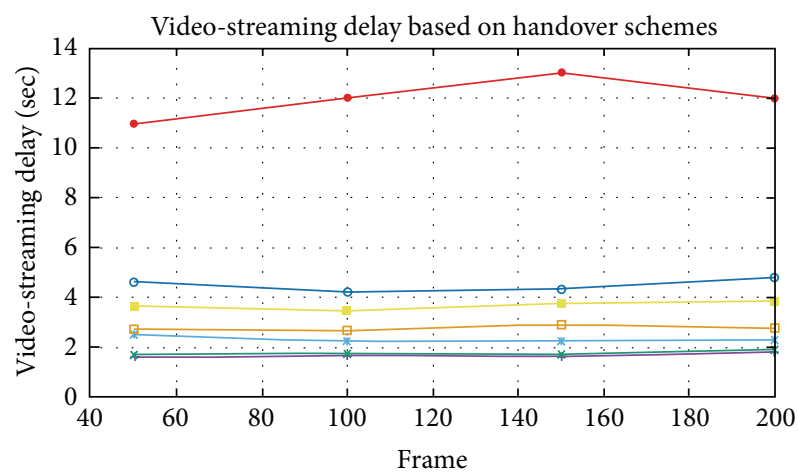

_ Video-streaming delay based on proposed scheme

$*$ Video-streaming delay based on HPIN

$\rightarrow$ * Video-streaming delay based on hybrid scheme

$\square$ Video-streaming delay based on eFMIPv6

-1-Video-streaming delay based on FMIPv6

$\rightarrow$ Video-streaming delay based on HMIPv6

$\rightarrow$ Video-streaming delay based on MIPv6

FIGURE 11: Video-streaming delay based on handover and forwarding schemes.

predicts a move to Area C. In this case, the handover succeeds if the MN actually hands over to Area C; otherwise, it fails. We first analyzed the handover-success probability $\left(P_{s}\right)$ and the handover-failure probability $\left(P_{f}\right)$ according to the $\mathrm{MN}$ initial state and based on the transition probability; then, we simulated the random-walk procedure with a sample size of 10,000 to verify the result of the analysis. $P_{s}$ can be calculated when the $\mathrm{MN}$ moves to one of the following states in Area C: State $(1,1,0)$, State $(0,1,0)$, State $(2,1,0)$, State $(1,1,1)$, State $(0,1,1)$, State $(2,1,1)$, State $(1,1,2)$, State $(0,1,2)$, and

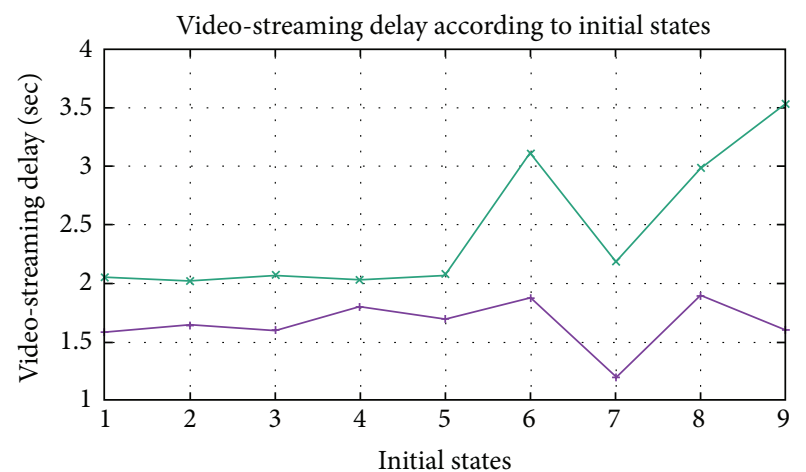

- Video-streaming delay based on proposed scheme * Video-streaming delay based on hybrid scheme

FIGURE 12: For the random-walk model, the video-streaming delay according to the initial states.

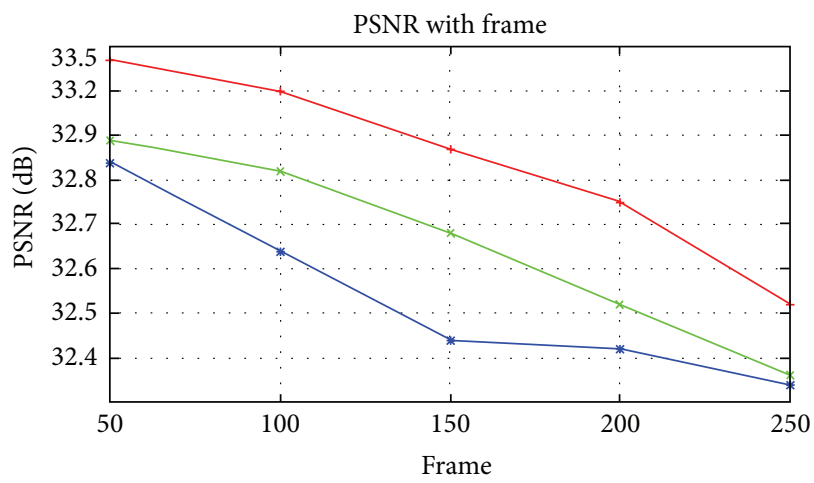

- Video streaming based on proposed scheme
- Video streaming based on eFMIPv6
$\rightarrow$ Video streaming based on MIPv6

FIGURE 13: Peak signal-to-noise ratio (PSNR) with frame.

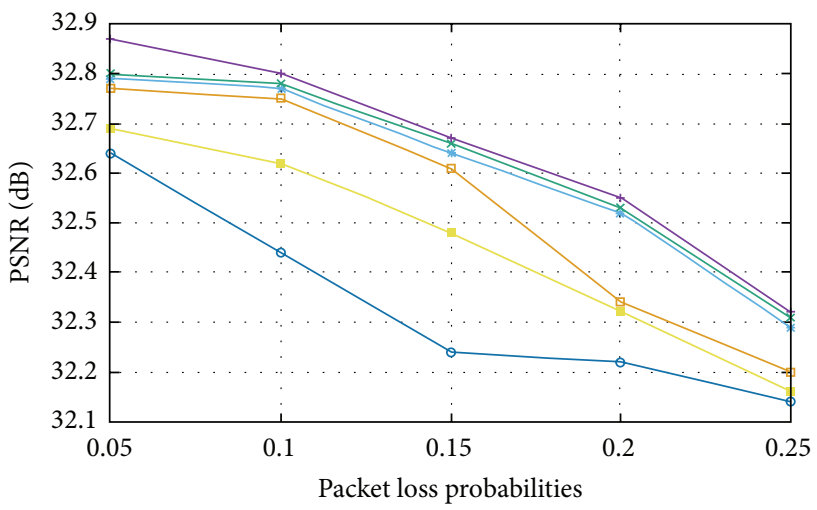

_ Video streaming based on the proposed scheme (MUX+DIV)

$\rightarrow$ Video streaming based on the proposed scheme (MUX)

\#-Video streaming based on the proposed scheme (DIV)

$\because$ Video streaming based on semisoft handover scheme [22]

—- Video streaming based on eFMIPv6

$\rightarrow$ Video streaming based on FMIPv6

FIGURE 14: Peak signal-to-noise ratio (PSNR) with packet loss probabilities. 


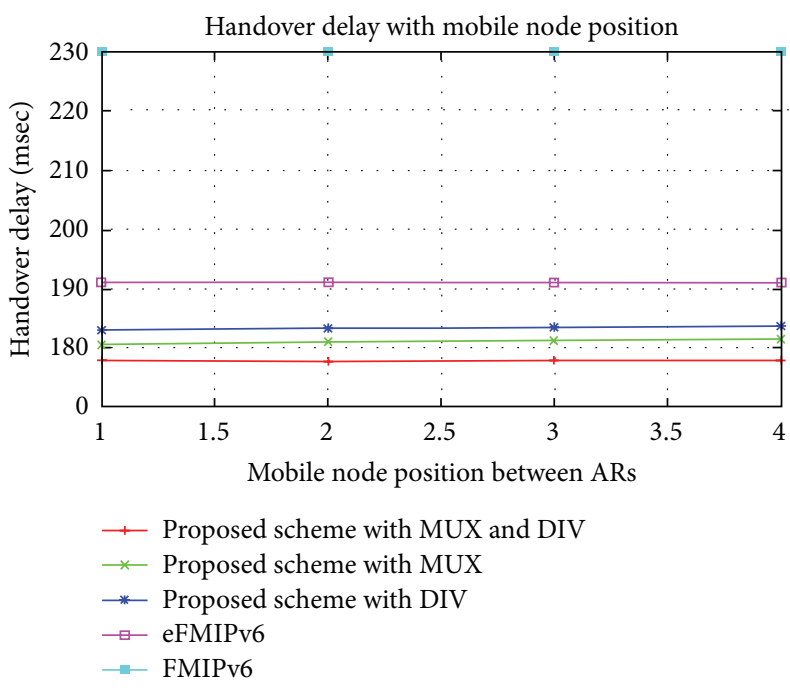

FIGURE 15: Handover delay with MN position.

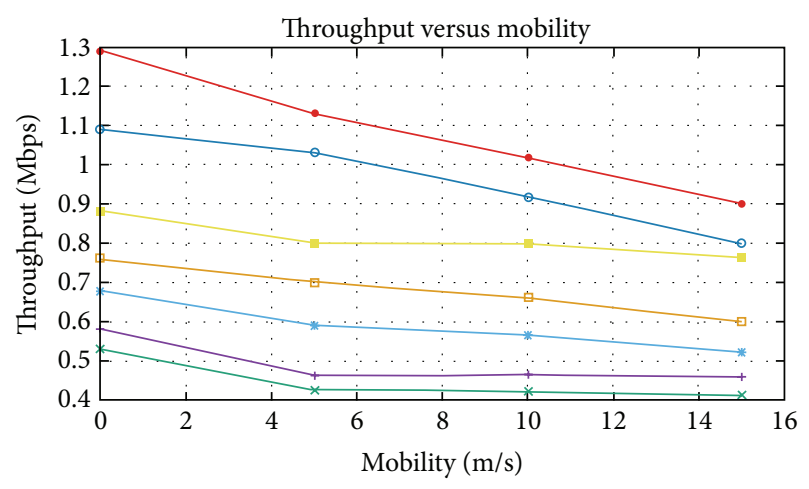

— Throughput based on HMIPv6

* Throughput based on MIPv6

* Throughput based on FMIPv6

$\rightarrow$ Throughput based on eFMIPv6

- Throughput based on hybrid scheme

$\rightarrow$ Throughput based on HPIN

- Throughput based on proposed scheme

Figure 16: Throughput.

TABLE 2: Complexity analysis.

State $(2,1,2) . P_{f}$ can be calculated when the MN performs handovers for areas other than Area C.

Figure 12 shows the video-streaming delays of our proposed scheme and the hybrid scheme according to the initial state; the initial states are possible locations for the MN. As expected, our scheme provides a delay that is shorter than that of the hybrid scheme because it is more robust in handover-failure situations under a dynamic-mobility environment, and it forwards the video packet quickly by using the proposed metric of (1).

Figures 13, 14, and 15 show that the proposed scheme achieves a higher PSNR and a lower handover latency with the MN position compared with the other schemes; that is, when the transmitted flows are mobile video streams, the proposed scheme selects the proper wireless network $w_{i}$ that comprises the temporal $\mathrm{CoAs}_{w_{i}}$ and the MIMO mode to minimize not only the handover delay but also the video delay. Figure 16 shows throughput gain of the proposed scheme with MIMO Tx techniques.

Additionally, Table 2 shows the complexity analysis of the proposed scheme and semisoft handover scheme inspects of graph theory. Actually, since our work is for each user's n-hop of multihop network with MUX scheme, complexity comparison with semisoft handover scheme seems to be ironic. $n$ means the number of users (nodes) in n-hop neighborhood MIMO network and $\mathrm{m}$ is the number of relations (edges) in n-hop neighborhood MIMO network. Also, $N$ means the number of every user (nodes) in whole MIMO network and $\mathrm{M}$ means the number of every relation (edges) in whole MIMO network.

Inspects of comparison analysis between the proposed scheme and semisoft handover scheme, our proposed scheme is seamless and practical with even partial information compared with the semisoft handover scheme with global information, since $n$ is the number of a few MUX capable nodes in the proposed scheme while $N$ is the number of total nodes of whole network in semisoft handover scheme.
TABte 2: Complexity analysis.

\begin{tabular}{lcc}
\hline & Semisoft handover [22] & Proposed scheme \\
\hline Time complexity & $O(N+M)$ & $O(n+m)$ \\
Space complexity & $O(N)$ & $O(n+m)$ \\
\hline
\end{tabular}

\section{Conclusion}

With the remarkable development of wireless technologies and mobile video streaming, the need to support a robust mobile-video-streaming service that is based on a seamless handover in MIMO-capable, heterogeneous wireless technologies continues to grow. In this paper, we consider robust mobile video streaming over several heterogeneous wireless networks, whereby the appropriate selection metric of the wireless technology with a verified tentative CoA and the MIMO mode are used at the MN to maximize video quality and minimize the video and handover delays for a robust mobile-video-streaming service. Through a performance evaluation that is based on an extended random-walk model for which the direction and speed of the MN are used, we show that our proposed scheme is a mechanism that is more robust than the other schemes for mobile video streaming.

The authors declare that there is no conflict of interests regarding the publication of this article.

\section{Acknowledgments}

This research was supported by the Basic Science Research Program through the National Research Foundation of Korea (NRF) that is funded by the Ministry of Science, ICT \& Future Planning (NRF-2014R1A1A1003562).

\section{Competing Interests}




\section{References}

[1] D. Gesbert, M. Shafi, D.-S. Shiu, P. J. Smith, and A. Naguib, "From theory to practice: an overview of MIMO space-time coded wireless systems," IEEE Journal on Selected Areas in Communications, vol. 21, no. 3, pp. 281-302, 2003.

[2] S. Aditya and S. Katti, "FlexCast: graceful wireless video streaming," in Proceedings of the 17th Annual International Conference on Mobile Computing and Networking (MobiCom '11), ACM, Las Vegas, Nev, USA, September 2011.

[3] S. Jakubczak and D. Katabi, MIT, SoftCast + Scalable Robust Mobile Video, ACM Mobicom, 2011.

[4] Cisco Visual Networking Index.

[5] H. Fathi, R. Prasad, and S. Chakraborty, "Mobility management for VoIP in 3G systems: evaluation of low-latency handoff schemes," IEEE Wireless Communications, vol. 12, no. 2, pp. 96104, 2005

[6] R. Koodli, "Fast handovers for mobile IPv6 (FMIPv6)," IETF, RFC 4068, 2005.

[7] D. B. Johnson, C. E. Perkins, and J. Arkko, "Mobility support in IPv6," RFC 3775, IETF, 2004.

[8] H. Soliman, C. Castelluccia, K. E. Malki, and L. Bellier, "Hierarchical Mobile IPv6 Mobility Management (HMIPv6)," IETF RFC 4140, 2005.

[9] S. Gundavelli, K. Leung, V. Devarapalli, K. Chowdhury, and B. Patil, "Proxy Mobile IPv6, RFC5213," August 2008.

[10] H. Oh, "A robust mobile video streaming in heterogeneous emerging wireless systems," KSII Transactions on Internet and Information Systems, 2012.

[11] H. Oh, J. Yoo, C.-K. Kim, and S. H. Ahn, "VMIPv6: a seamless and robust vehicular MIPv6 for vehicular wireless networks and vehicular intelligent transportation systems (V-Winet/V-ITS)," Journal of Information Science and Engineering, vol. 26, no. 3, pp. 833-850, 2010.

[12] H. Oh, K. Yoo, J. Na, and C. Kim, "A seamless handover scheme in IPv6-based mobile networks," International Journal of Ad Hoc and Ubiquitous Computing, vol. 4, no. 1, pp. 54-60, 2009.

[13] H. Oh, K. Yoo, J. Na, and C.-K. Kim, "A robust seamless handover scheme for the support of multimedia services in heterogeneous emerging wireless networks," Wireless Personal Communications, vol. 52, no. 3, pp. 593-613, 2010.

[14] A. M. Vegni and E. Natalizio, "A hybrid (N/M)CHO soft/hard vertical handover technique for heterogeneous wireless networks," Ad Hoc Networks, vol. 14, pp. 51-70, 2014.

[15] C. Makaya and S. Pierre, "An architecture for seamless mobility support in IP-based next-generation wireless networks," IEEE Transactions on Vehicular Technology, vol. 57, no. 2, pp. 12091225, 2008.

[16] K. Sundaresan and R. Sivakumar, "Routing in ad-hoc networks with MIMO links," in Proceedings of the 13th IEEE International Conference on Network Protocols (ICNP '05), pp. 85-95, IEEE, Boston, Mass, USA, November 2005.

[17] J.-S. Wang and J.-S. Sheu, "Study of handover techniques for 4G network MIMO systems," in Proceedings of the International Conference on Electronics, Signal Processing and Communication Systems, 2013.

[18] S. Cho and J.-W. Choi, "Downlink soft handover using multicell MIMO for 4G-LTE smart-phones," in Proceedings of the IEEE International Conference on Consumer Electronics (ICCE '14), pp. 207-208, Las Vegas, Nev, USA, January 2014.
[19] H. Lee, H. Lee, and D.-H. Cho, "Novel handover decision method in wireless communication systems with multiple antennas," in Proceedings of the IEEE 64th Vehicular Technology Conference (VTC '06), pp. 2099-2103, Montreal, Canada, September 2006.

[20] V. Jungnickel, K. Manolakis, L. Thiele, T. Wirth, and T. Haustein, "Handover sequences for interference-aware transmission in multicell MIMO networks," in Proceedings of the International ITG Workshop on Smart Antennas (WSA '09), February 2009.

[21] S. A. Kravchuk, "Research of influence of multiantenna technology MIMO on realisation of handover in cellular radioaccess systems," in Proceedings of the 21st International Crimean Conference on Microwave \& Telecommunication Technology (CriMiCo '11), pp. 353-354, Sevastopol, Ukraine, September 2011.

[22] J.-T. Lim and D.-H. Cho, "Gain analysis of adaptive MIMO semisoft handover in LTE-OFDM systems," in Proceedings of the IEEE 81st Vehicular Technology Conference (VTC Spring '15), pp. 1-5, Glasgow, UK, May 2015.

[23] B. Megha and Y. S. Lalitha, "Downlink capacity improvement for LTE using multi-cell MIMO during handover," International Journal of Innovative Research in Science, Engineering and Technology, 2015.

[24] Y.-H. Han, J. Choi, and S.-H. Hwang, "Reactive handover optimization in IPv6-based mobile networks," IEEE Journal on Selected Areas in Communications, vol. 24, no. 9, pp. 1758-1772, 2006.

[25] J.-T. Park and S.-M. Chun, "QoS-guaranteed IP mobility management for fast-moving vehicles with multiple network interfaces," IET Communications, vol. 6, no. 15, pp. 2287-2295, 2012.

[26] Q. B. Mussabbir, W. Yao, Z. Niu, and X. Fu, "Optimized FMIPv6 using IEEE 802.21 MIH services in vehicular networks," IEEE Transactions on Vehicular Technology, vol. 56, no. 6, pp. 33973407, 2007.

[27] H. Fathi, S. S. Chakraborty, and R. Prasad, "Optimization of mobile IPv6-based handovers to support VoIP services in wireless heterogeneous networks," IEEE Transactions on Vehicular Technology, vol. 56, no. 1, pp. 260-270, 2007.

[28] D. Salomon, Guide to Data Compression Methods, Springer, Berlin, Germany, 2002.

[29] PSNR, https://en.wikipedia.org/wiki/Peak_signal-to-noise_ratio.

[30] A. J. Chan, A. Pande, E. Baik, and P. Mohapatra, "Temporal quality assessment for mobile videos," in Proceedings of the 18th Annual International Conference on Mobile Computing and Networking (Mobicom '12), pp. 221-232, Istanbul, Turkey, 2012.

[31] Y. Y. An, B. H. Yae, K. W. Lee, Y. Z. Cho, and W. Y. Jung, "Reduction of handover latency using MIH services in MIPv6," in Proceedings of the 20th International Conference on Advanced Information Networking and Applications (AINA '06), pp. 229234, IEEE, Vienna, Austria, April 2006.

[32] YUV Sequence, http://trace.eas.asu.edu/yuv/.

[33] T. Camp, J. Boleng, and V. Davies, "A survey of mobility models for ad hoc network research," Wireless Communications and Mobile Computing, vol. 2, no. 5, pp. 483-502, 2002. 


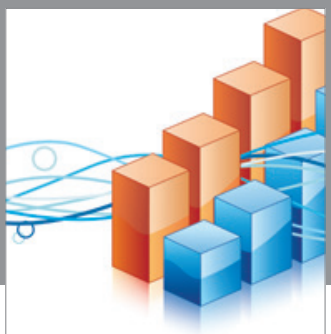

Advances in

Operations Research

vatem alat4

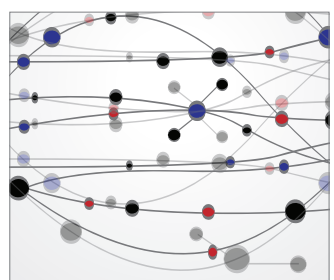

\section{The Scientific} World Journal
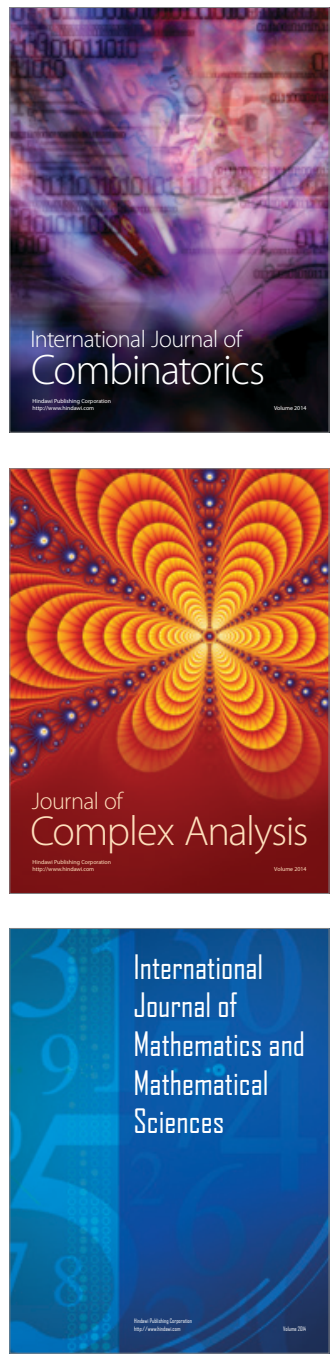
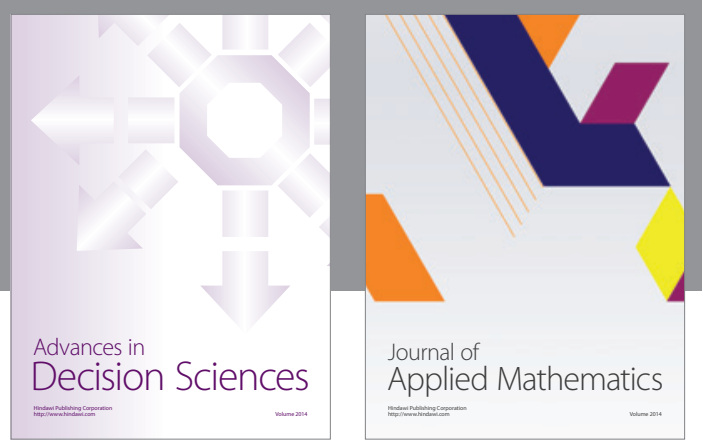

Algebra

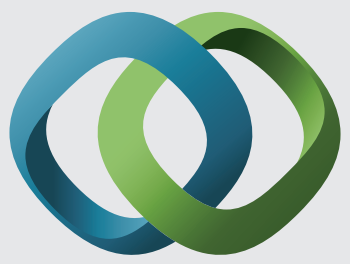

\section{Hindawi}

Submit your manuscripts at

http://www.hindawi.com
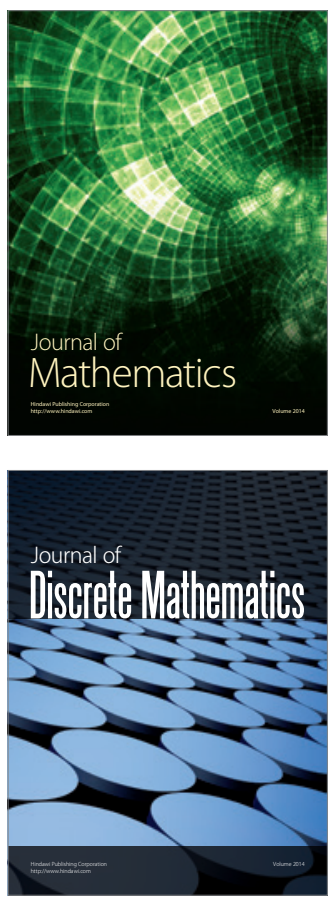

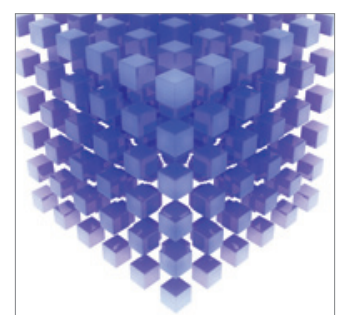

Mathematical Problems in Engineering
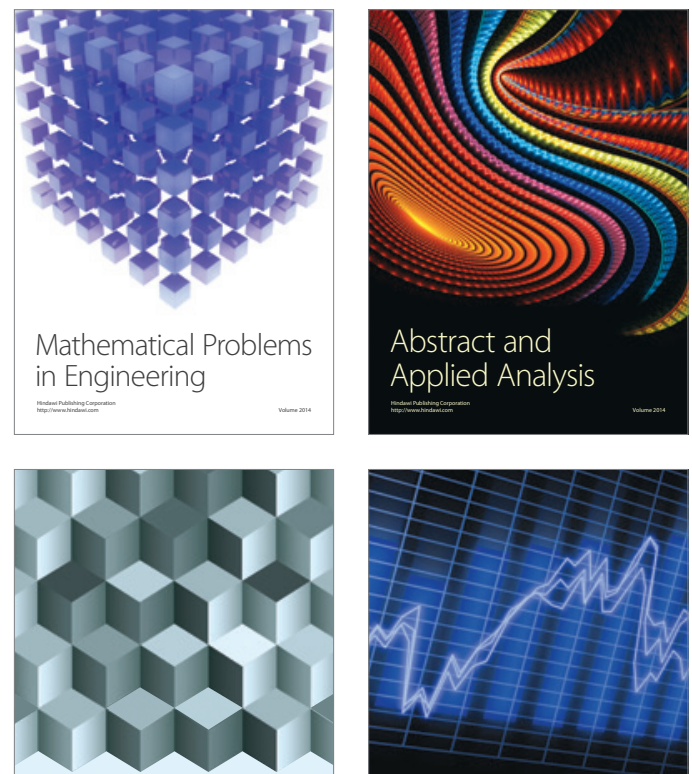

Journal of

Function Spaces

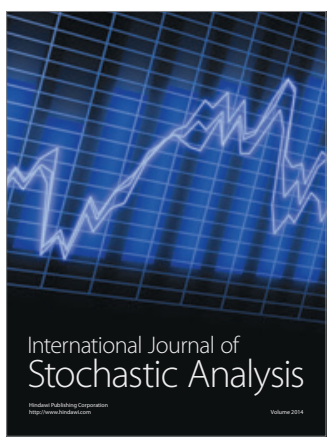

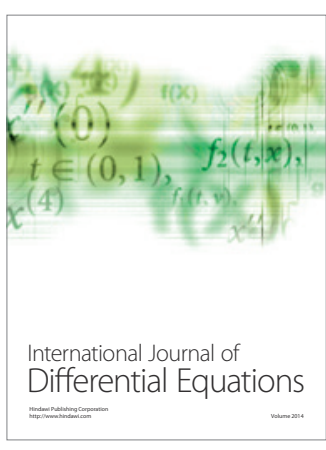
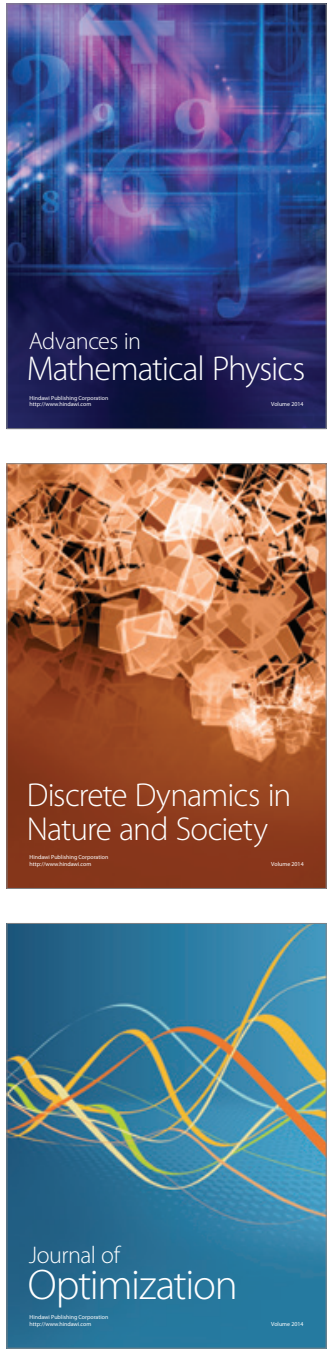